\title{
The role of delta and theta oscillations during ego-motion in healthy adult volunteers
}

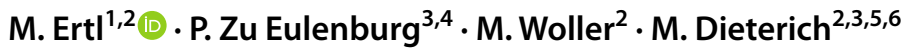

Received: 18 August 2020 / Accepted: 30 December 2020 / Published online: 3 February 2021

(c) The Author(s) 2021

\begin{abstract}
The successful cortical processing of multisensory input typically requires the integration of data represented in different reference systems to perform many fundamental tasks, such as bipedal locomotion. Animal studies have provided insights into the integration processes performed by the neocortex and have identified region specific tuning curves for different reference frames during ego-motion. Yet, there remains almost no data on this topic in humans.

In this study, an experiment originally performed in animal research with the aim to identify brain regions modulated by the position of the head and eyes relative to a translational ego-motion was adapted for humans. Subjects sitting on a motion platform were accelerated along a translational pathway with either eyes and head aligned or a $20^{\circ}$ yaw-plane offset relative to the motion direction while EEG was recorded.

Using a distributed source localization approach, it was found that activity in area PFm, a part of Brodmann area 40, was modulated by the congruency of translational motion direction, eye, and head position. In addition, an asymmetry between the hemispheres in the opercular-insular region was observed during the cortical processing of the vestibular input. A frequency specific analysis revealed that low-frequency oscillations in the delta- and theta-band are modulated by vestibular stimulation. Source-localization estimated that the observed low-frequency oscillations are generated by vestibular core-regions, such as the parieto-opercular region and frontal areas like the mid-orbital gyrus and the medial frontal gyrus.
\end{abstract}

Keywords Vestibular system · Vestibular stimulation · Reference frames · Hemispheric asymmetry $\cdot$ Alpha activity · Passive motion $\cdot$ Multisensory

Communicated by Bill J Yates.

M. Ertl

matthias.ertl@psy.unibe.ch

1 Department of Psychology, University of Bern, Fabrikstrasse 8, 3012 Bern, Switzerland

2 Department of Neurology, Ludwig-Maximilians-Universität München, München, Germany

3 German Center for Vertigo and Balance Disorders (IFBLMU), Ludwig-Maximilians-Universität München, München, Germany

4 Institute for Neuroradiology, Ludwig-Maximilians-Universität München, München, Germany

5 Graduate School of Systemic Neuroscience, Ludwig-Maximilians-Universität München, München, Germany

6 Munich Cluster for Systems Neurology (SyNergy), Munich, Germany

\section{Introduction}

The vestibular system is known to interact highly with other sensory systems, such as the visual, proprioceptive, and somatosensory systems. This integration of multimodal information enables humans to perform sophisticated tasks, such as bipedal upright stance, locomotion, and the coordination of eye and head movements during active or passive body motion (St George and Fitzpatrick 2011; Dieterich and Brandt 2015a). While much of the processing and integration of vestibular input already takes place on a subcortical level, vestibular input is also transferred to, and processed by, the neocortex. Such vestibular projections have been found to feed into higher cognitive functions like spatial navigation (Yoder and Taube 2009; Zwergal et al. 2015) and egocentric 3-D space referencing (Brandt et al. 2014; Hummel et al. 2016; Lopez 2016; Ellis et al. 2017).

While functional neuroimaging (zu Eulenburg et al. 2012; Lopez et al. 2012) and lesion studies (Dieterich and 
Brandt 2015b) have identified the cortical network involved in the processing of vestibular input, electroencephalography (EEG) studies (McNerney et al. 2011; Todd et al. 2014, 2016; Kammermeier et al. 2015; Ertl et al. 2017) provided insight into the temporal dynamics of these processes. The excellent temporal resolution of EEG enabled the identification of early, middle and long latency potentials and their association with particular brain structures. Additionally, initial attempts to analyze and interpret vestibular-evoked oscillation patterns have been made (Bertora and Bergmann 1995; Gale et al. 2016; Ertl et al. 2017, 2020).

The majority of neuroimaging and EEG studies have focused exclusively on the vestibular system or on low-level integration processes. However, there is evidence that information provided by the vestibular system also significantly contributes to cognitive tasks (Brandt et al. 2014; Dennis et al. 2017; Ellis et al. 2017; Popp et al. 2017). For example, spatial navigation in a complex world forces the brain to simultaneously process information provided by multiple sensory systems and only the successful integration of all sensory input enables an efficient interaction with the environment using a multimodal representation of space and objects (Andersen et al. 1997). This task becomes additionally challenging since the various sensory systems use different reference frames. For example, it is assumed that the vestibular input by means of head acceleration or a change in the position relative to the gravitational field of the earth is represented in a head-centered reference frame (Chen et al. 2013a). The visual system, however, acquires the raw data from the retina which results in an eye-centered reference frame. The various reference systems and the reconciliation between systems with different reference frames have been the topic of multiple animal studies (Brotchie et al. 1995; Snyder et al. 1998; Chen et al. 2013a). In fact, one study in monkeys associated the ventral intraparietal area (VIP) with a body (or world)-centered reference frame, the medial superior temporal area (MSTd) with an eye- or headcentered reference frame and the parietoinsular vestibular cortex (PIVC) with an ambivalent head-body-centered reference frame (Chen et al. 2013b). It is likely that a similar set of reference frames exists in humans.

Over the last two decades multiple human neuroimaging studies have reported a reciprocal interaction between the vestibular and the visual cortex (Brandt et al. 1998, 2002; Deutschländer et al. 2002; Dieterich et al. 2003; DellaJustina et al. 2014). Although some of the studies should be interpreted with caution since the sample sizes were small (Brandt et al. 1998; Bense et al. 2001; Inoue et al. 2014) and results were not always corrected properly for multiple testing (Deutschländer et al. 2002), the finding of a visual-vestibular interaction seems robust. Notably, a recent study on multisensory interaction using near infrared caloric vestibular stimulation failed to find a reciprocal visual-vestibular interaction (Ferrè et al. 2015). Nevertheless, a reciprocal inhibitory visual-vestibular interaction is a reasonable assumption as it provides an efficient mechanism for shifting weights between incoming data streams from different sensory systems during multisensory integration (Brandt et al. 1998). In EEG, the occipital alpha rhythm can be considered a good surrogate marker for the activity of the visual cortex. It is well-established that alpha activity over occipital regions is most dominant in the absence of visual input and decreases as visual input is processed. Within this model, strong alpha activity is a marker of an idle status of the visual cortex. By accepting this model, occipital alpha becomes a useful tool for investigation of visual-vestibular interaction.

At this point, only very few EEG studies addressing naturalistic motion processing in humans exist. Most of the knowledge on vestibular cortical processing relies on artificial stimulation in fMRI or PET experiments. For this reason, many aspects, for example, on temporal interactions between brain regions, which are well studied in other sensory systems are largely unknown. The presented publication reports new insights relevant to the field.

We aimed to identify brain regions modulated by the position of the head and eyes relative to a translational body movement. Furthermore, we aimed to find evidence for a reciprocal visual-vestibular interaction as reported by functional magnetic resonance imaging (fMRI) and positron emission tomography (PET) studies in the EEG alpha activity.

\section{Methods}

\section{Subjects}

The EEG of 30 healthy subjects was recorded. Due to artifacts, seven subjects have been removed from the analysis. The analyzed group (12 female, 11 male) had a mean age of 25.3 years $(\mathrm{SD}=5.0$ years). Twenty-one subjects were $100 \%$ right handed and two were $100 \%$ left-handed according to the 10-item inventory of the Edinburgh test (Oldfield 1971). All participants declared that they had no prior history of neurological or neuro-otological disease. All subjects gave their informed written consent and were paid for participation. The study was approved by the local ethics committee (Munich).

\section{Stimuli and procedure}

The experiment was designed to match a previously published study in non-human primates (Chen et al. 2013b). The subjects were seated in a racing chair mounted on a 6-degreeof-freedom motion platform (Moog@ 6DOF2000E). Motion 
platforms enable the stimulation of the vestibular system with naturalistic passive whole-body accelerations while recording EEG signals (Ertl and Boegle 2019). To mask motion correlated noise produced by the motion of the platform, white noise of $90 \mathrm{~dB}$ (SPL) was presented via noisecancelling headphones during the experiment. The visual targets were displayed on a $102 \times 57 \mathrm{~cm}$ flat screen (JVC GD-463D10) mounted on, and therefore moving with, the platform. Additionally, subjects wore goggles (EyeSeeTec, Munich Germany) equipped with a laser diode centered between the eyes. The subject's head was fixed to the chair. Since the task required voluntary head movements, the fixation was not as restrictive as in previous experiments, thus allowing for rotations around the yaw axis.

The task was designed to investigate brain processes related to input with congruent and incongruent reference frames. During the experiment, subjects were asked to position their head and gaze according to visual stimuli presented on the screen (Fig. 1). Two different targets (eye and head) occurred either on the center of the screen or at $\mathrm{a} \pm 20^{\circ}$ angle in the yaw plane. Every trial started with the occurrence of an "+" on the screen (i.e. head target). The subjects were asked to turn the head-fixed laser dot onto this target and keep this position until the end of the trial. One second later, an additional "+" occurred on the screen (i.e. eye target). Subjects were asked to fixate the " $X$ " with their eyes without moving the head away from the " $X$ ". One second after, the " $X$ " occurred on the screen the platform performed a transversal movement. After the movement, the platform remained still for $1.5 \mathrm{~s}$ before returning to the start position. Participants were instructed to keep their eyes and head on the target until they perceive the platform moving back. Five different conditions were tested during the experiment. In the congruent condition, the head position, the eye position, and the platform movement were aligned (AS). In another condition, the movement, eye and head positions differed all by $20^{\circ}(\mathrm{AD})$. Furthermore, three conditions in which only one system (eye (ED), head (HD), movement (MD)) differed from the two other were performed. The order of the conditions was randomized. Every condition occurred 12 times per block.

The experiment consisted of 240 trials ( 48 per condition) which were grouped into four blocks of 60 trials. The breaks between the blocks were between 2 and $5 \mathrm{~min}$. The entire experiment took about $45 \mathrm{~min}$.

\section{EEG recording and preprocessing}

Throughout the experiment, EEG was continuously recorded using 32 active electrodes mounted on a cap (EASYCAP, Woerthsee-Etterschlag, Germany) according to the international 10/20 system. The FCz electrode was used as the reference. The data were acquired at a sampling rate of
1000 samples per second. Impedances were kept below 5 $\mathrm{k} \Omega$. Additionally, the platform motion was tracked using two accelerometers. The signals of the accelerometer and the EEG electrodes were recorded using the Brain Vision Recorder (Version 1.20). A band-pass $(0.016-1000 \mathrm{~Hz}$, $6 \mathrm{~dB} /$ Octave and $30 \mathrm{~dB} /$ Octave) filter was applied to the EEG data.

The data were preprocessed using the Brain Vision Analyzer (Version 2.1). At first, the data were band-pass filtered (0.1-45 Hz, $48 \mathrm{~dB} /$ Octave) and down-sampled to $250 \mathrm{sam}$ ples per second. Data were re-referenced to common average reference $(\mathrm{CAR})$ and the old reference $(\mathrm{FCz})$ was recovered as regular channel. An independent component analysis (ICA) was performed and components reflecting any kind of artifacts, particularly eye blinks, muscle, or motion artifacts were identified by a visual inspection of the time courses and topographies. These components were removed from the data before the back projection (Jung et al. 2000; Hoffmann and Falkenstein 2008).

The time traces of the acceleration sensors and the logfiles generated during the experiment were analyzed using an in-house program, implemented in Matlab (MathWorks), and markers indicating condition and movement onset were written onto the EEG data sets.

\section{Event related potentials (ERP)}

The preprocessed data were segmented from -500 to $3000 \mathrm{~ms}$ relative to the onset of the platform movements as detected by the acceleration sensors. This allows investigation of the following $500 \mathrm{~ms}$ baseline before the movement, a period of $\sim 1750 \mathrm{~ms}$ while the subject is moved and a $1250 \mathrm{~ms}$ period after the movement (Fig. 2a). The segments were baseline corrected with respect to the activity in the $500 \mathrm{~ms}$ pre-movement baseline. Averages were calculated for the five conditions and additionally across all conditions (Fig. 2b).

\section{Time-frequency analysis}

To study the temporal dynamics of evoked oscillations, a wavelet analysis was performed on the segments as created for the ERP analysis. The frequency space between 1 and $45 \mathrm{~Hz}$ was analyzed using linear steps and a complex MorletWavelet. The Morlet parameter was set to 4 . The output was $\mathrm{z}$-Transformed ( -500 to $0 \mathrm{~ms}$ ). Transformations were calculated for all subjects. Grand averages for all five conditions and one including all trials were calculated. A visual inspection of the time-frequency plots revealed that power changes were most prominent in the delta and theta band (Fig. 2c). This was confirmed by frequency-band wise comparisons of the average power between baseline and the stimulus interval. Further analyses were therefore only performed for the 

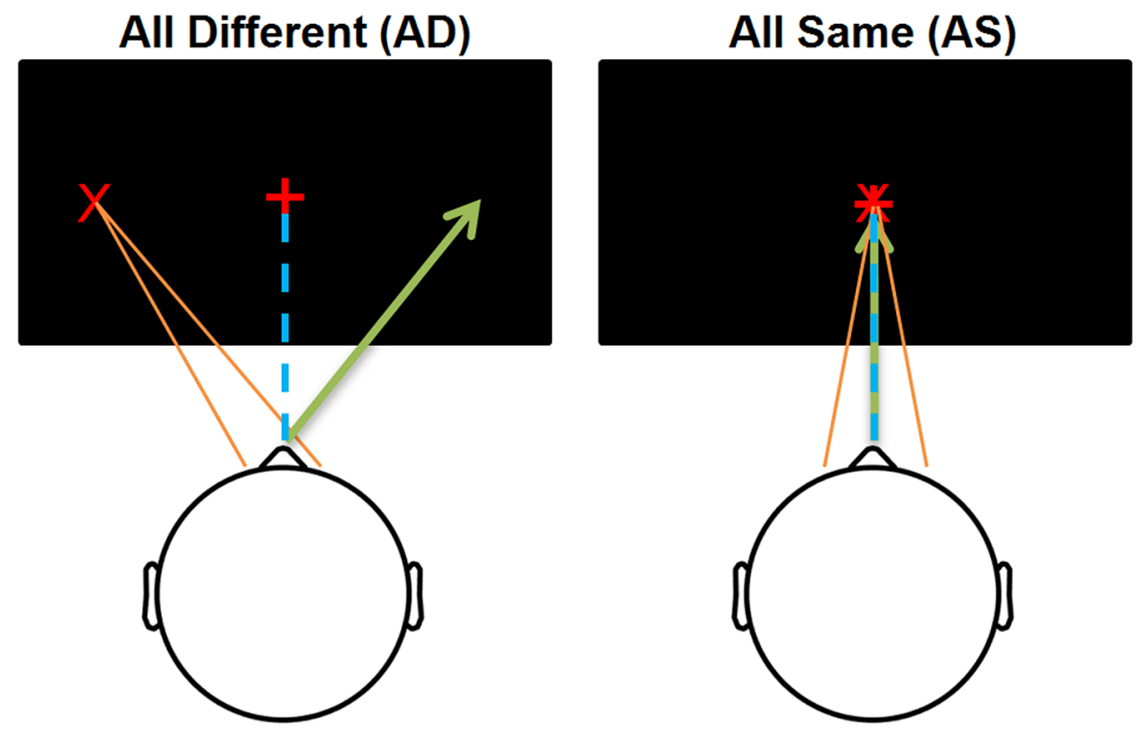

\section{$X$ eye target \\ + head target \\ - movement direction \\ - head direction \\ - gaze direction}
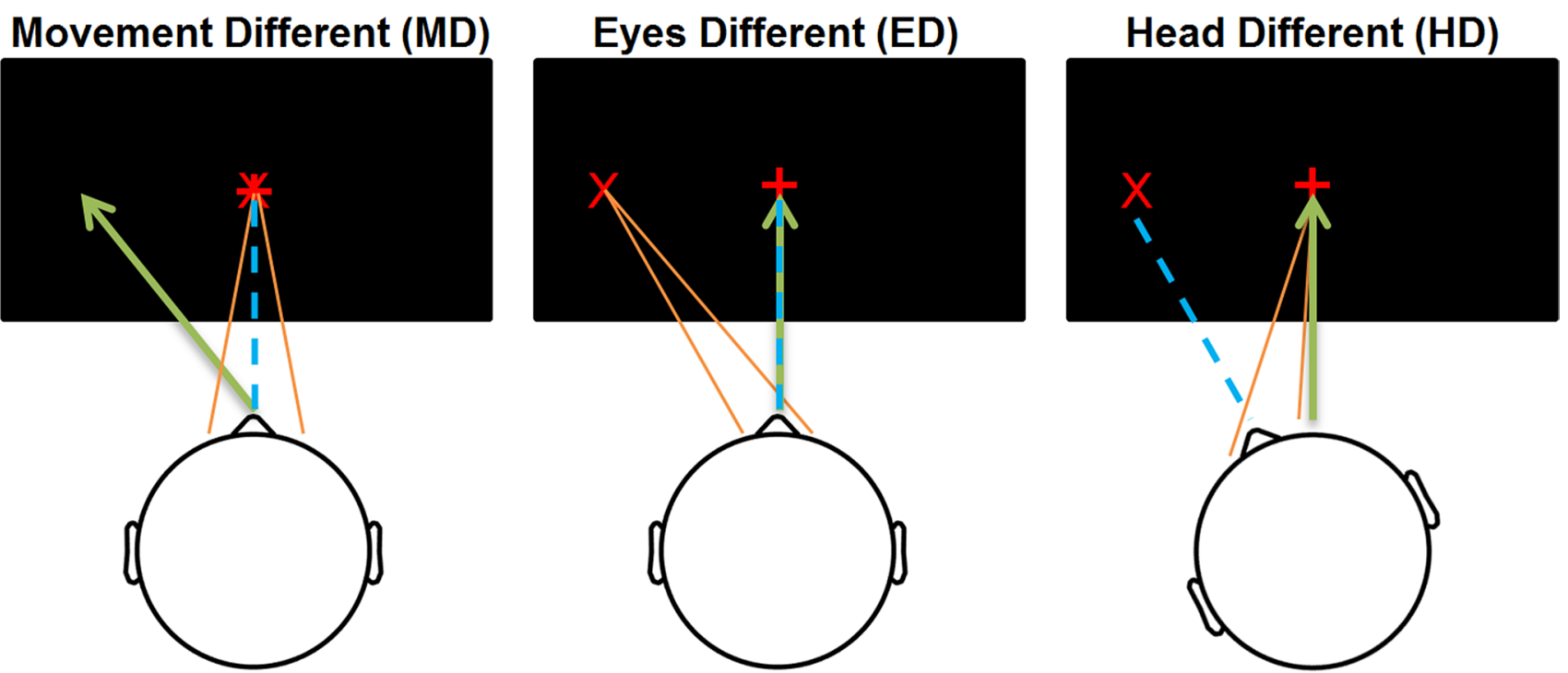

Fig. 1 Visualization of the head, and eye position relative to the platform movement during the five conditions. In the "all different" (AD) condition the head-, eye, and movement all pointed into three different directions with an angle of $20^{\circ}$ between them. During the "all same" (AS) condition the eyes, head, and movement directions were all aligned. During the other three conditions either the movement (MD), eyes (ED), head (HD) positions deviated from the remaining two. The positions of the targets varied between trials and all possible combinations were presented during the experiment

a.htm) implementation by Pacual-Marqui (Pascual-Marqui 2002, 2007). eLORETA (exact low resolution brain electromagnetic tomography algorithm) is an extended and optimized weighted minimum norm-based approach with the property of exact localization to the test point sources, albeit with a relatively low spatial resolution. LORETA is one of the most commonly used algorithms for distributed source localization. It has been verified multiple times by comparing it to results of PET and fMRI studies (Dierks et al. 2000; Vitacco et al. 2002; Pizzagalli et al. 2003).

The average time courses ranging from -500 to $3000 \mathrm{~ms}$ relative to motion onset were exported to LOERTA and 

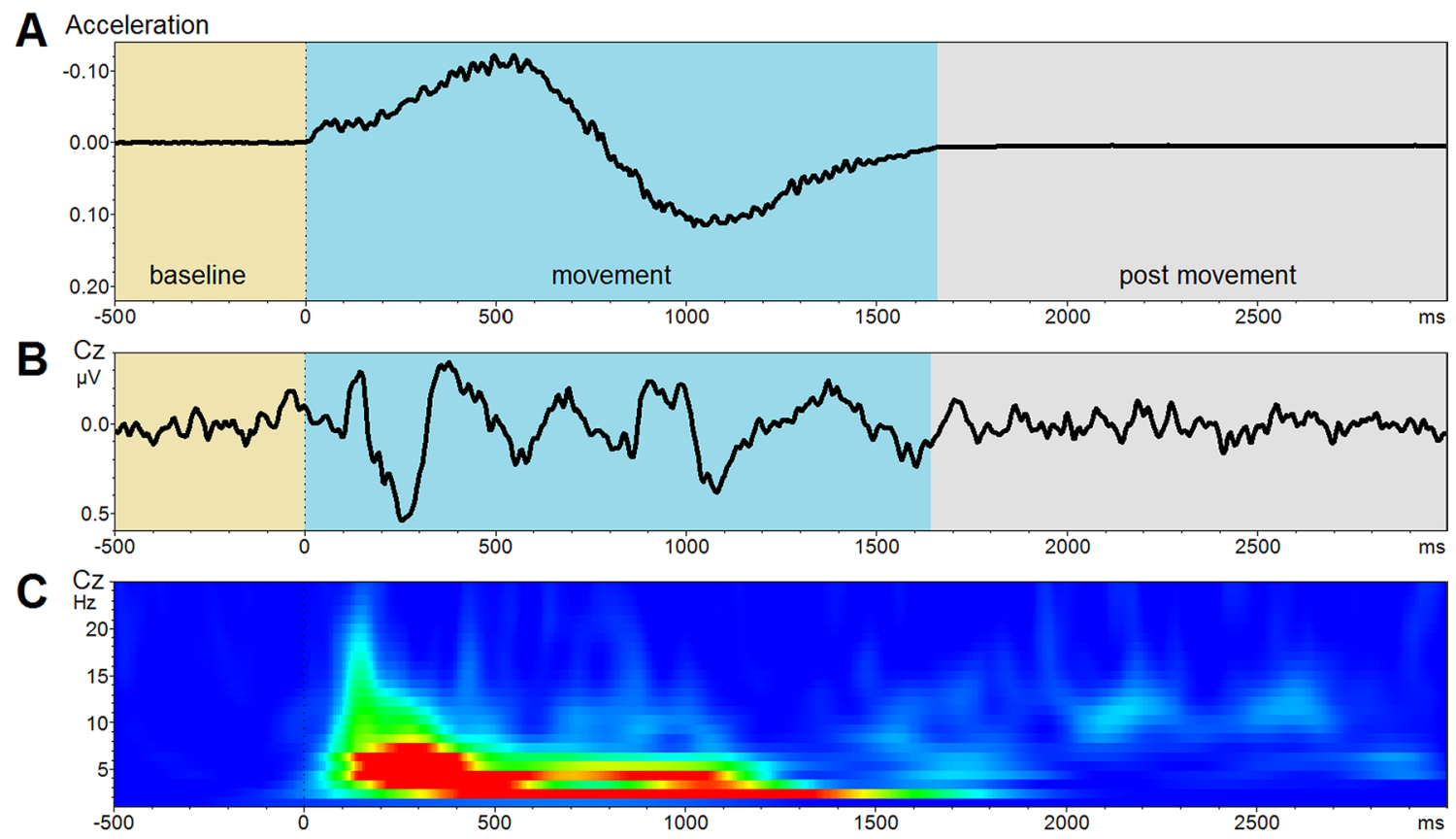

Fig. 2 a Acceleration profile used for this experiment. b Grand Average of all conditions and subjects. Due to different vestibular thresholds across the subjects and due to a less restrictive head fixation the VestEPs described by other studies (P1, N1, P2) are less pronounced. c Time-frequency representation of the time course. A strong signal increase during the movement can be observed in the delta $(1-4 \mathrm{~Hz})$ and theta-band $(4-7 \mathrm{~Hz})$

time-varying cross-spectra were then frequency-wise transformed into the source space. Paired t-tests were performed comparing the mean activity at baseline with the mean activity during the platform motion.

The identified regions were labeled based on cytoarchitectonic maps (Caspers et al. 2006; Malikovic et al. 2007) and functional imaging data as implemented in the SPM anatomy toolbox (Eickhoff et al. 2006). For area CSv, which is not part of the anatomy toolbox, we compared our results with the coordinates reported in previous studies (Smith et al. 2012; Ertl et al. 2017).

We also addressed the question of a hemispheric asymmetry between the left and right opercular-insular region/ PIVC as reported earlier by fMRI studies (Dieterich et al. 2003; Janzen et al. 2008). Therefore, we extracted the activity during all motion periods from two ROIs created based on the activation pattern observed for the contrast MotionRest (Fig. 3a). The average activity in the left and right PIVC was then compared using Wilcoxon rank-sum test.

\section{Visual-vestibular interaction (Alpha-power)}

Occipital alpha power may be used as a surrogate marker for the activity in the visual cortex and for subsequent analysis of visual-vestibular interaction. To compare the alpha power at electrode $\mathrm{Oz}$ during vestibular stimulation and rest periods, a Fourier Transformation was calculated for any of the Gaussian window function with a width of 20 frames. The 

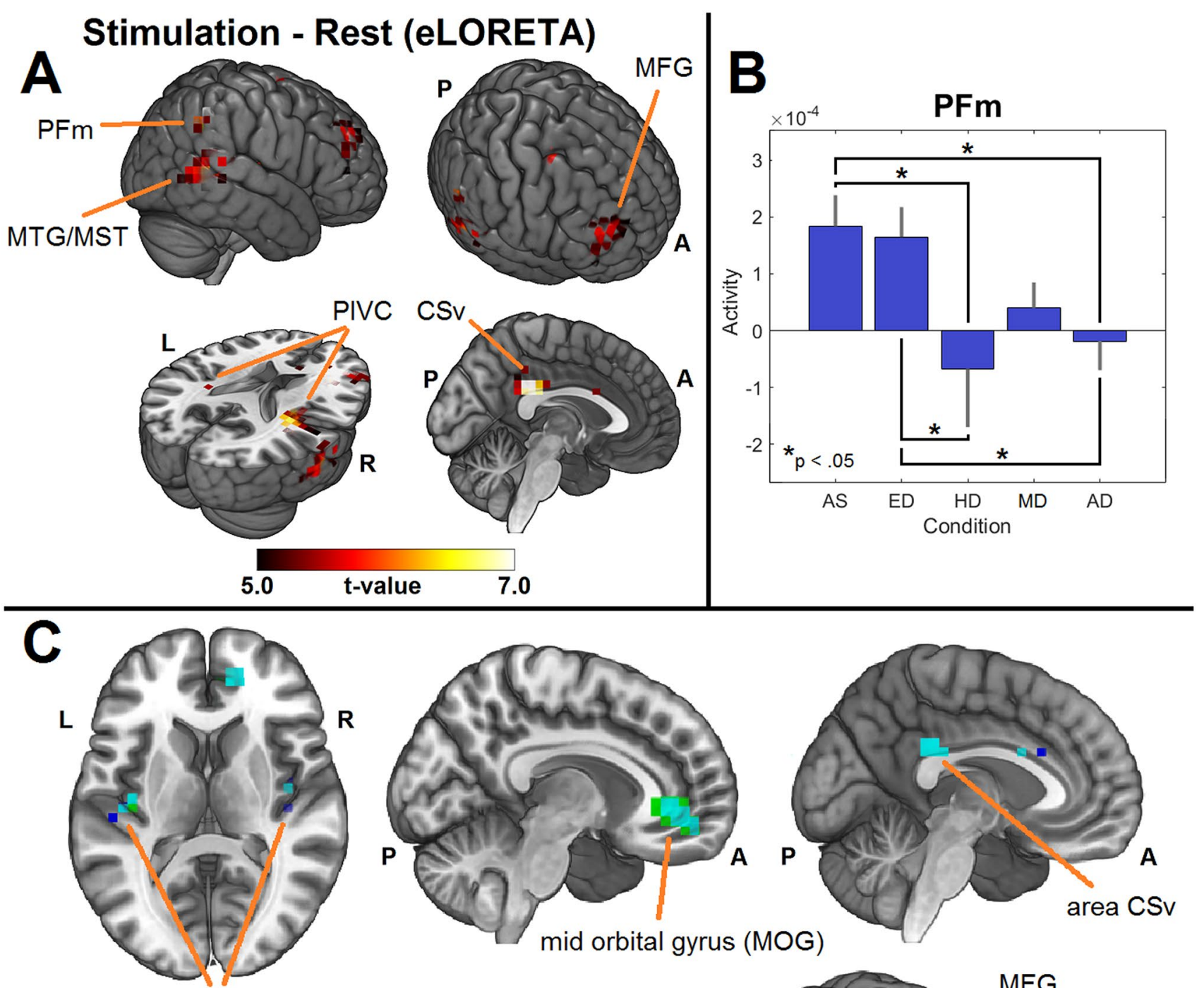

opercular insular region (PIVC)
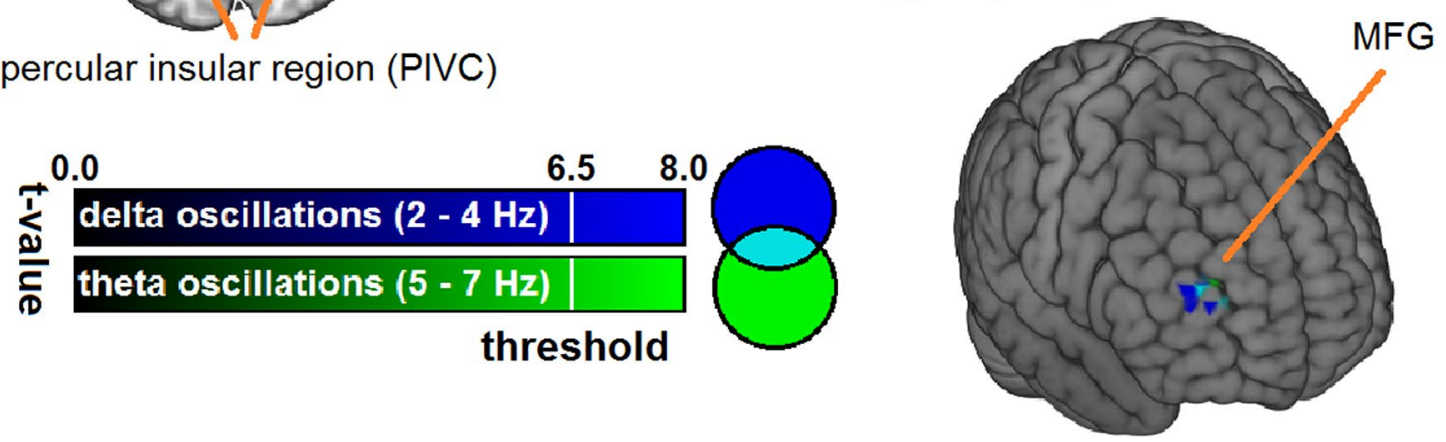

Fig. 3 a Results of the source localization analysis. We found a network consisting of the opercular-insular cortex (PIVC), area CSv, the middle temporal gyrus (MTG), the medial frontal gyrus (MFG) and area PFm, a sub-region of Brodmann area 40, as main generators during the movement compared to the rest period. Comparing the activity in left and right PIVC a hemispheric asymmetry with stronger activity in the right hemisphere can be seen. b Only the activity in area PFm was significantly modulated by the relative positions of the eyes, head, and movement. The activity was particularly decreased in conditions in which the head was not aligned with the eyes and movement. c Frequency-specific source localization analysis for frequencies in the delta- and theta-band comparing the activity during motion to the rest period. The results revealed activity in the cortical vestibular "core network" (PIVC, area CSv) and frontal structures (MOG, MFG) 
$1.5 \mathrm{~s}$ stimulation and the following $1.5 \mathrm{~s}$ rest interval. The alpha-band was defined as frequencies between 8 and $12 \mathrm{~Hz}$. At first, t-tests were calculated between the conditions on a single subject level for the mean activity in the alpha-band. In a second analysis, the single-trial data were averaged for every subject and a $t$-test was calculated at the group level.

\section{Results}

\section{Event-related potentials (ERP)}

During the movement period, a series of positive and negative components were observable at the scalp level (Fig. 2b). The first components showed the P1/N1/P2 pattern as described in previous vestibular ERP studies (Todd et al. 2016; Ertl et al. 2017, 2020). Contrary to our previous experiments, the ERPs elicited by the used stimulation in the current study were, due to different vestibular thresholds, not phase-locked. We therefore abstained from a systematic comparison of amplitudes and latencies across conditions.

\section{Time-frequency analysis}

A comparison of the delta and theta power during the three periods baseline, movement, and post-movement (rest), revealed significant increases in low-frequency oscillations during the movements (Fig. 2c). Delta and theta power during movement was increased relative to baseline (delta: $t=4.15, p<0.001$; theta: $t=6.67, p<0.001)$ and the postmovement period (delta: $t=3.94, p<0.001$; theta: $t=5.50$, $p<0.001)$. A comparison between baseline and the postmovement period revealed an increased theta power in the post-movement period (delta: $t=0.76, p=0.45$; theta: $t=2.58, p<0.05)$. The alpha power was increased relative to the pre-stimulus $(p<0.001)$ but not the post-stimulus interval $(p=0.189)$. The beta and gamma power were not significantly altered compared to the pre-stimulus (beta: $p=0.010$; gamma $p=0.411$ ) or the post-stimulus period (beta: $p=0.758$; gamma $p=0.867$ ).

Comparing the delta and theta power across the five conditions using an ANOVA did not reveal any significant differences (delta: $\mathrm{F}=0.67, p=0.61$; theta: $\mathrm{F}=0.37, p=0.83$ ).

\section{Distributed source localization}

The contrast between the movement and the baseline interval revealed the known vestibular network as source of the evoked activity. In particular area CSv, the posterior opercular-insular region, Brodmann area 40 (BA 40) with peak activity in area PFm, the medial temporal gyrus including area MST and the medial frontal gyrus, were identified as main sources (Fig. 3a). The systematic comparison of the evoked activity in the ROIs revealed significant results for BA 40 only. A substantial difference was found for the contrast AS-AD $(p=0.014)$. Additionally, the contrasts AS-HD $(p=0.029)$, AD-ED $(p=0.035)$ and ED-HD $(\mathrm{p}=0.045)$ showed significant differences of evoked activity in BA 40. In short, significant differences were only found in conditions where the head was not aligned with the eyes and movement (Fig. 3b). Comparison of the activity of the left and right opercular-insular region/PIVC during all motion intervals showed significantly $(p<0.001)$ stronger activity in the right hemisphere.

The frequency-specific source localization revealed a network of sources as generators of the delta and theta oscillations (Fig. 3c). Both frequency bands had sources in the left and right opercular-insular region, the mid-orbital gyrus (MOG) and the medial frontal gyrus (MFG). Theta oscillations showed additional sources in area CSv and the anterior cingulate gyrus.

\section{Visual-vestibular interaction (Alpha-power)}

The comparison of alpha power on the group level did not reveal significant differences between stimulation and rest periods (Fig. 4). Comparing the alpha power between the rest and stimulation conditions individually, we found significantly $(p<0.05)$ increased alpha power in the rest condition in three subjects. No subject showed significantly decreased alpha power.

\section{Discussion}

In this study, we investigated the impact of head and eye position relative to the movement direction of the body. The most active areas during vestibular stimulation by means of distributed source localization were area $\mathrm{CSv}$, the posterior opercular-insular region, BA 40, the medial temporal gyrus and the medial frontal gyrus. These results are similar to the estimated generators observed in recent experiments on the same motion platform using short translational accelerations (Ertl et al. 2017, 2020) as well as data acquired by other neuroimaging modalities (Zu Eulenburg et al. 2012; Lopez et al. 2012). A region of interest analysis of our data revealed that only the signal in a sub-region of BA 40 was modulated by the task. A time-frequency analysis of the evoked oscillations showed a significant power increase during the vestibular stimulation which was generated by the opercular-insular region, the mid-orbital gyrus, and the MFG. Additionally, sources in area CSv and the anterior cingulate cortex (ACC) were only found for theta oscillations.

Area PFm is one out of five cytoarchitectonically defined sub-regions of the supramarginal gyrus (Caspers et al. 2006) and part of BA 40 which has been associated 
Fig. 4 Fourier-Transformation of the signal recorded at electrode $\mathrm{Oz}$ for frequencies between 0 and $20 \mathrm{~Hz}$ during the rest period and the vestibular stimulation (Vest) elicited by the platform movement. Comparing the alpha power for the individual alpha-frequencies of the subjects a slight but not significant decrease can be observed during motion compared to rest
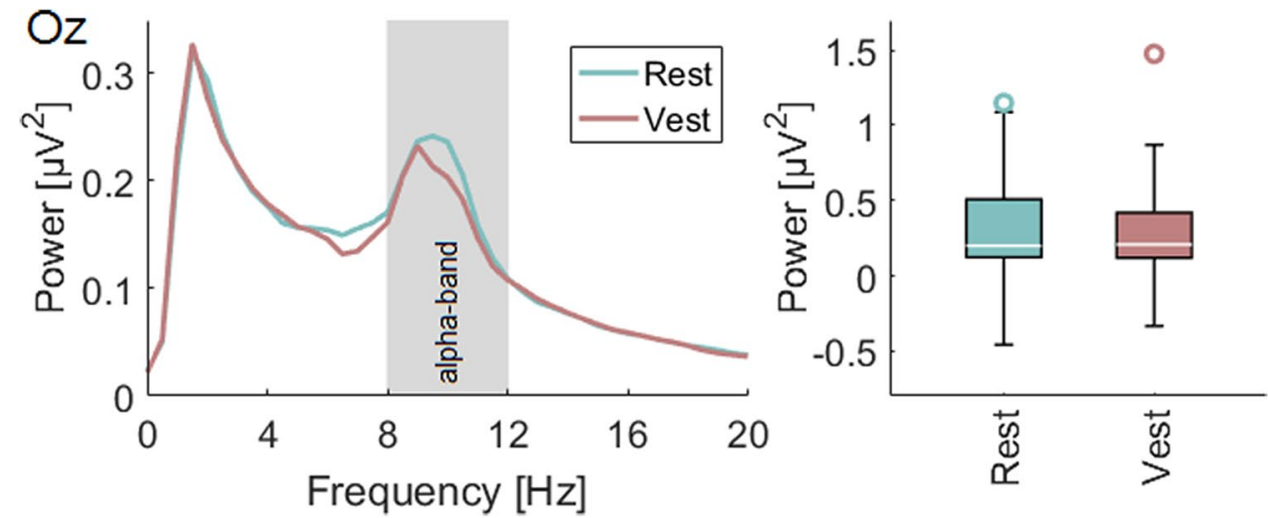

with spatial attention and re-orienting tasks (Corbetta et al. 2008; Caspers et al. 2013). Activity in BA 40 has also been reported by imaging studies on the vestibular system (Deutschländer et al. 2002; Dieterich et al. 2003; Janzen et al. 2008; Della-Justina et al. 2014) and is known from animal studies to receive vestibular input (Guldin and Grüsser 1998). Nevertheless, its functional role within the vestibular cortical network is not yet clear. Here, we found decreased activity in BA 40 with the peak in area PFm during conditions in which the head position was not aligned with the eye position or the movement direction. This finding might provide an important puzzle piece in future models of the functional role of BA 40 during vestibular stimulation.

The comparison of the overall activity in the left and right opercular-insular region showed a significantly pronounced activity in the right hemisphere in right-handers (Fig. 3a). This asymmetry between the hemispheres is a common finding during vestibular stimulation (Dieterich et al. 2003; Janzen et al. 2008) and was here confirmed using a further methodical approach.

Using an electrophysiological measurement, the analysis of the occipital alpha activity during and after vestibular stimulation confirmed the results of previous neuroimaging studies regarding visual-vestibular interactions (Brandt et al. 1998; Della-Justina et al. 2014). Alpha activity was found to be increased, although not significantly so, during rest periods as compared to periods with vestibular stimulation. Participants in the current study performed a fixation task during body movements, i.e., a combined visual and vestibular stimulation. This fixation likely reduced the alpha power detectable at occipital electrodes compared to an eyes closed condition. In analogy, we speculate that a comparison between vestibular stimulation and rest with closed eyes would lead to a more pronounced difference between the conditions and we aim to address this question in future studies.

The time-frequency analysis showed the strongest effects in the delta and theta-band. A power increase in the delta band has already been reported by stimulation of the horizontal canal by a rotatory chair (Bertora and Bergmann 1995). An increase in theta-band power with the onset of semicircular canal stimulation has also been demonstrated in healthy subjects and patients with bilateral vestibular loss (Gale et al. 2016). Both studies used stimulations with durations greater than one second. This appeared to be a relevant parameter as we had used significantly shorter acceleration profiles and failed to show changes in low frequencies due to otolith stimulation (Ertl et al. 2017). The finding of lowfrequency changes in the present study may suggest that a minimum stimulation duration is necessary to evoke delta/ theta oscillations. Since, the delta and theta power was not found to differ significantly between the five conditions, we argue that low-frequency oscillations are related to the perceived acceleration rather than to the relative head and eye position. As the frequency of the used acceleration profile was lower than $1 \mathrm{~Hz}$, and therefore not within the analyzed frequency range, it is unlikely that the observed delta and theta effects are technical artifacts caused by the platform or movements of the subjects. The source localization results of the low-frequency oscillations in the delta and theta band suggest that low frequencies reflect the interaction between parts of the vestibular "core" network (PIVC, CSv) and frontal structures (MFG, MOG). Frontal theta oscillations in primates are assumed to reflect active operations during high-level cognitive processes and realizing the need for top-down control (Cavanagh and Frank 2014). This idea is supported by reports of frontal theta in studies on action monitoring (Cavanagh et al. 2012; Cohen 2016), multisensory attention and integration (Friese et al. 2016; Yan et al. 2016), emotion regulation (Ertl et al. 2013) and working memory (Hsieh and Ranganath 2014; Maurer et al. 2015).

In this manuscript, we presented data demonstrating a contribution of frontal structures to the processing of vestibular input by means of translational accelerations. A distributed source localization analysis revealed that the vestibular "core network" and the identified frontal regions (MFG, MOG) showed increased delta and theta activity during motion. Future studies should address this co-activation 
in more depth, for example, using more complex visual stimuli that can be consistent or inconsistent with the perceived vestibular stimulation.

Supplementary Information The online version contains supplementary material available at https://doi.org/10.1007/s00221-020-06030-3.

Funding Open Access funding provided by Universität Bern. The work was supported by the BMBF (German Federal Ministry of EducationBMBF 01 EO 1401), the Graduate School of Neuroscience, and the German Foundation for Neurology (Deutsche Stiftung Neurologie).

Data availability The datasets generated during and/or analysed during the current study are available from the corresponding author on reasonable request.

\section{Compliance with ethical standards}

Conflict of interest The authors declare that they have no conflict of interest.

Ethics approval All participants completed an informed consent form before performing the experiments. The study was approved by the by the local ethics committee of the medical faculty of the LudwigMaximilians Universität München, Germany.

Open Access This article is licensed under a Creative Commons Attribution 4.0 International License, which permits use, sharing, adaptation, distribution and reproduction in any medium or format, as long as you give appropriate credit to the original author(s) and the source, provide a link to the Creative Commons licence, and indicate if changes were made. The images or other third party material in this article are included in the article's Creative Commons licence, unless indicated otherwise in a credit line to the material. If material is not included in the article's Creative Commons licence and your intended use is not permitted by statutory regulation or exceeds the permitted use, you will need to obtain permission directly from the copyright holder. To view a copy of this licence, visit http://creativecommons.org/licenses/by/4.0/.

\section{References}

Andersen RA, Snyder LH, Bradley DC, Xing J (1997) Multimodal representation of space in the posterior parietal cortex and its use in planning movements. Annu Rev Neurosci 20:303-330. https:// doi.org/10.1146/annurev.neuro.20.1.303

Bense S, Stephan T, Yousry TA et al (2001) Multisensory cortical signal increases and decreases during vestibular galvanic stimulation (fMRI). J Neurophysiol 85:886-899

Bertora GO, Bergmann JM (1995) Cortical responses of vestibular reactions measured by topographic brain mapping and vestibular evoked potentials. Acta Otolaryngol 115:126-129. https://doi. org/10.3109/00016489509125208

Brandt T, Bartenstein P, Janek A, Dieterich M (1998) Reciprocal inhibitory visual-vestibular interaction-Visual motion stimulation deactivates the parieto-insular vestibular cortex. Brain 121:1749-1758. https://doi.org/10.1093/brain/121.9.1749

Brandt T, Glasauer S, Stephan T et al (2002) Visual-vestibular and visuovisual cortical interaction: New insights from fMRI and PET. Ann N Y Acad Sci 956:230-241. https://doi. org/10.1111/j.1749-6632.2002.tb02822.x
Brandt T, Strupp M, Dieterich M (2014) Towards a concept of disorders of "higher vestibular function." Front Integr Neurosci 8:1-8. https://doi.org/10.3389/fnint.2014.00047

Brotchie PR, Andersen RA, Snyder LH, Goodman SJ (1995) Head position signals used by parietal neurons to encode locations of visual stimuli. Nature 375:232-235. https://doi. org/10.1038/375232a0

Caspers S, Geyer S, Schleicher A et al (2006) The human inferior parietal cortex: Cytoarchitectonic parcellation and interindividual variability. Neuroimage 33:430-448. https://doi. org/10.1016/j.neuroimage.2006.06.054

Caspers S, Schleicher A, Bacha-Trams M et al (2013) Organization of the human inferior parietal lobule based on receptor architectonics. Cereb Cortex 23:615-628. https://doi.org/10.1093/ cercor/bhs048

Cavanagh JF, Frank MJ (2014) Frontal theta as a mechanism for cognitive control. Trends Cogn Sci 18:414-421. https://doi. org/10.1016/j.tics.2014.04.012

Cavanagh JF, Zambrano-Vazquez L, Allen JJB (2012) Theta lingua franca: A common mid-frontal substrate for action monitoring processes. Psychophysiology 49:220-238. https://doi.org/10.1 111/j.1469-8986.2011.01293.x

Chen X, DeAngelis GC, Angelaki DE (2013a) Eye-centered representation of optic flow tuning in the ventral intraparietal area. J Neurosci 33:18574-18582. https://doi.org/10.1523/jneur osci.2837-13.2013

Chen X, DeAngelis GC, Angelaki DE (2013b) Diverse spatial reference frames of vestibular signals in parietal cortex. Neuron 80:1310-1321. https://doi.org/10.1016/j.neuron.2013.09.006

Cohen MX (2016) Midfrontal theta tracks action monitoring over multiple interactive time scales. Neuroimage 141:262-272. https://doi.org/10.1016/j.neuroimage.2016.07.054

Corbetta M, Patel G, Shulman GL (2008) The reorienting system of the human brain: from environment to theory of mind. Neuron 58:306-324. https://doi.org/10.1016/j.neuron.2008.04.017

Della-Justina HM, Gamba HR, Lukasova K et al (2014) Interaction of brain areas of visual and vestibular simultaneous activity with fMRI. Exp Brain Res 233:237-252. https://doi. org/10.1007/s00221-014-4107-6

Dennis EL, Rashid F, Ellis MU et al (2017) Diverging white matter trajectories in children after traumatic brain injury. Neurology 88:1392-1399. https://doi.org/10.1212/WNL.000000000000380 8

Deutschländer A, Bense S, Stephan T et al (2002) Sensory system interactions during simultaneous vestibular and visual stimulation in PET. Hum Brain Mapp 16:92-103. https://doi.org/10.1002/ hbm. 10030

Dierks T, Jelic V, Pascual-Marqui RD et al (2000) Spatial pattern of cerebral glucose metabolism (PET) correlates with localization of intracerebral EEG-generators in Alzheimer's disease. Clin Neurophysiol 111:1817-1824. https://doi.org/10.1016/S1388 -2457(00)00427-2

Dieterich M, Brandt T (2015a) The bilateral central vestibular system: its pathways, functions, and disorders. Ann N Y Acad Sci 1343:10-26. https://doi.org/10.1111/nyas. 12585

Dieterich M, Brandt T (2015) Why acute unilateral vestibular cortex lesions mostly manifest without vertigo. Neurology $84: 1-5$

Dieterich M, Bense S, Lutz S et al (2003) Dominance for vestibular cortical function in the non-dominant hemisphere. Cereb Cortex 13:994-1007

Eickhoff SB, Weiss PH, Amunts K et al (2006) Identifying human parieto-insular vestibular cortex using fMRI and cytoarchitectonic mapping. Hum Brain Mapp 27:611-621. https://doi.org/10.1002/ hbm.20205 
Ellis AW, Klaus MP, Mast FW (2017) Vestibular cognition: the effect of prior belief on vestibular perceptual decision making. J Neurol 264:74-80. https://doi.org/10.1007/s00415-017-8471-6

Ertl M, Boegle R (2019) Investigating the vestibular system using modern imaging techniques-A review on the available stimulation and imaging methods. J Neurosci Methods. https://doi. org/10.1016/j.jneumeth.2019.108363

Ertl M, Hildebrandt M, Ourina K et al (2013) Emotion regulation by cognitive reappraisal - the role of frontal theta oscillations. Neuroimage 81:412-421. https://doi.org/10.1016/j.neuroimage .2013.05.044

Ertl M, Moser M, Boegle R et al (2017) The cortical spatiotemporal correlate of otolith stimulation: Vestibular evoked potentials by body translations. Neuroimage 155:50-59. https://doi. org/10.1016/j.neuroimage.2017.02.044

Ertl M, Klaus M, Mast FW et al (2020) Spectral fingerprints of correct vestibular discrimination of the intensity of body accelerations. Neuroimage 219:117015. https://doi.org/10.1016/j.neuro image.2020.117015

Ferrè ER, Walther LE, Haggard P (2015) Multisensory interactions between vestibular, visual and somatosensory signals. PLoS ONE 10:1-16. https://doi.org/10.1371/journal.pone.0124573

Friese U, Daume J, Göschl F et al (2016) Oscillatory brain activity during multisensory attention reflects activation, disinhibition, and cognitive control. Sci Rep 6:1-11. https://doi.org/10.1038/ srep32775

Gale S, Prsa M, Schurger A et al (2016) Oscillatory neural responses evoked by natural vestibular stimuli in humans. J Neurophysiol 115:1228-1242. https://doi.org/10.1152/jn.00153.2015

Goldman RI, Stern JM, Engel J, Cohen MS (2002) Simultaneous EEG and fMRI of the alpha rhythm. NeuroReport 13:24872492. https://doi.org/10.1097/00001756-200212200-00022

Guldin WO, Grüsser O-J (1998) Is there a vestibular cortex? Trends Neurosci 21:254-259

Gutteling TP, Medendorp WP (2016) Role of alpha-band oscillations in spatial updating across whole body motion. Front Psychol 7:1-12. https://doi.org/10.3389/fpsyg.2016.00671

Gutteling TP, Selen LPJ, Medendorp WP (2015) Parallax-sensitive remapping of visual space in occipito-parietal alpha-band activity during whole-body motion. J Neurophysiol 113:1574-1584. https://doi.org/10.1152/jn.00477.2014

Gutteling TP, Schutter DJLG, Medendorp WP (2017) Alpha-band transcranial alternating current stimulation modulates precision, but not gain during whole-body spatial updating. Neuropsychologia 106:52-59. https://doi.org/10.1016/j.neuropsychologi a.2017.09.005

Hoffmann S, Falkenstein M (2008) The correction of eye blink artefacts in the EEG: A comparison of two prominent methods. PLoS ONE. https://doi.org/10.1371/journal.pone.0003004

Hsieh L-T, Ranganath C (2014) Frontal Midline Theta Oscillations during Working Memory Maintenance and Episodic Encoding and Retrieval. Neuroimage 15:1-7. https://doi.org/10.1038/ jid.2014.371

Hummel N, Cuturi LF, MacNeilage PR, Flanagin VL (2016) The effect of supine body position on human heading perception. $\mathrm{J}$ Vis 16:1-11. https://doi.org/10.1167/16.3.19

Inoue N, Fukuda S, Inada T et al (2014) Effect of ibudilast on the reciprocal inhibitory visual-vestibular interaction closely related to dizziness after cerebral ischemia. J Stroke Cerebrovasc Dis 23:51-55. https://doi.org/10.1016/j.jstrokecerebrovasdis .2012 .09 .007

Janzen J, Schlindwein P, Bense S et al (2008) Neural correlates of hemispheric dominance and ipsilaterality within the vestibular system. Neuroimage 42:1508-1518. https://doi.org/10.1016/j. neuroimage.2008.06.026
Jung T, Makeig S, Humphries C et al (2000) Removing Electroencephalographic aretfacts by blind source seperation. Psychophysiology 37:163-178. https://doi.org/10.1111/14698986.3720163

Kammermeier S, Singh A, Noachtar S et al (2015) Intermediate latency evoked potentials of cortical multimodal vestibular areas: Acoustic stimulation. Clin Neurophysiol 126:614-625. https://doi. org/10.1016/j.clinph.2014.06.036

Laufs H, Kleinschmidt A, Beyerle A et al (2003) EEG-correlated fMRI of human alpha activity. Neuroimage 19:1463-1476. https ://doi.org/10.1016/S1053-8119(03)00286-6

Liu Z, de Zwart JA, Yao B et al (2012) Finding thalamic BOLD correlates to posterior alpha EEG. Neuroimage 63:1060-1069. https://doi.org/10.1016/j.neuroimage.2012.08.025

Lopez C (2016) The vestibular system: balancing more than just the body. Curr Opin Neurol 29:74-83

Lopez C, Blanke O, Mast FW (2012) The human vestibular cortex revealed by coordinate-based activation likelihood estimation meta-analysis. Neuroscience 212:159-179. https://doi. org/10.1016/j.neuroscience.2012.03.028

Malikovic A, Amunts K, Schleicher A et al (2007) Cytoarchitectonic analysis of the human extrastriate cortex in the region of V5/ MT+: A probabilistic, stereotaxic map of area hOc5. Cereb Cortex 17:562-574. https://doi.org/10.1093/cercor/bhj181

Maurer U, Brem S, Liechti M et al (2015) Frontal midline theta reflects individual task performance in a working memory task. Brain Topogr 28:127-134. https://doi.org/10.1007/s 1054 8-014-0361-y

McNerney KM, Lockwood AH, Lou CM et al (2011) Use of 64-channel electroencephalography to study neural otolithevoked responses. J Am Acad Audiol 22:143-155. https://doi. org/10.3766/jaaa.22.3.3

Moosmann M, Ritter P, Krastel I et al (2003) Correlates of alpha rhythm in functional magnetic resonance imaging and near infrared spectroscopy. Neuroimage 20:145-158. https://doi. org/10.1016/S1053-8119(03)00344-6

Oldfield RC (1971) The assessment and analysis of handedness: The Edinburgh inventory. Neuropsychologia 9:97-113. https://doi. org/10.1016/0028-3932(71)90067-4

Pascual-Marqui RD (2002) Standardized low resolution brain electromagnetic tomography (sLORETA): technical details. Methods Find Exp Clin Pharmacol 24D:5-12

Pascual-Marqui RD (2007) Discrete, 3D distributed, linear imaging methods of electric neuronal activity. Part 1: exact, zero error localization. http://arxiv.org/pdf/07103341 1-16

Pizzagalli DA, Oakes TR, Davidson RJ (2003) Coupling of theta activity and glucose metabolism in the human rostral anterior cingulate cortex: An EEG/PET study of normal and depressed subjects. Psychophysiology 40:939-949. https://doi. org/10.1111/1469-8986.00112

Popp P, Wulff M, Finke K et al (2017) Cognitive deficits in patients with a chronic vestibular failure. J Neurol. https://doi.org/10.1007/ s00415-016-8386-7

Smith AT, Wall MB, Thilo KV (2012) Vestibular inputs to human motion-sensitive visual cortex. Cereb Cortex 22:1068-1077. https ://doi.org/10.1093/cercor/bhr179

Snyder LH, Grieve KL, Brotchie P, Andersen R, a, (1998) Separate body- and world-referenced representations of visual space in parietal cortex. Nature 394:887-891. https://doi.org/10.1038/29777

St George RJ, Fitzpatrick RC (2011) The sense of self-motion, orientation and balance explored by vestibular stimulation. J Physiol 589:807-813. https://doi.org/10.1113/jphysiol.2010.197665

Todd NPM, Paillard AC, Kluk K et al (2014) Source analysis of short and long latency vestibular-evoked potentials (VsEPs) produced by left vs. right ear air-conducted $500 \mathrm{~Hz}$ tone pips. Hear Res 312:91-102. https://doi.org/10.1016/j.heares.2014.03.006 
Todd NPM, Govender S, Colebatch JG (2016) Vestibular-dependent inter-stimulus interval effects on sound evoked potentials of central origin. Hear Res 341:190-201. https://doi.org/10.1016/j.heare s.2016.07.017

Vitacco D, Brandeis D, Pascual-Marqui R, Martin E (2002) Correspondence of event-related potential tomography and functional magnetic resonance imaging during language processing. Hum Brain Mapp 17:4-12. https://doi.org/10.1002/hbm.10038

Yan T, Bi X, Zhang M et al (2016) Age-related oscillatory theta modulation of multisensory integration in frontocentral regions. Neuroreport 27:796-801

Yoder RM, Taube JS (2009) Head direction cell activity in mice: Robust directional signal depends on intact otolith organs. J Neurosci 29:1061-1076. https://doi.org/10.1523/jneurosci.1679-08.2009
Zu Eulenburg P, Caspers S, Roski C, Eickhoff SB (2012) Meta-analytical definition and functional connectivity of the human vestibular cortex. Neuroimage 60:162-169. https://doi.org/10.1016/j.neuro image.2011.12.032

Zwergal A, Schöberl F, Xiong G et al (2015) Anisotropy of Human Horizontal and Vertical Navigation in Real Space: Behavioral and PET Correlates. Cereb Cortex 26:4392-4404. https://doi. org/10.1093/cercor/bhv213

Publisher's Note Springer Nature remains neutral with regard to jurisdictional claims in published maps and institutional affiliations. 